\title{
Seismoelectric Fluid/Porous-Medium Interface Response Model and Measurements
}

\author{
M. D. Schakel • D. M. J. Smeulders • E. C. Slob • \\ H. K. J. Heller
}

Received: 31 January 2011 / Accepted: 30 August 2011 / Published online: 30 September 2011

(C) The Author(s) 2011. This article is published with open access at Springerlink.com

\begin{abstract}
Coupled seismic and electromagnetic (EM) wave effects in fluid-saturated porous media are measured since decades. However, direct comparisons between theoretical seismoelectric wavefields and measurements are scarce. A seismoelectric full-waveform numerical model is developed, which predicts both the fluid pressure and the electric wavefields in a fluid in which a porous disc is embedded. An experimental setup, in which pressure and electric signals in the fluid are simultaneously measured, is presented. The setup allows the detection of the EM field that is generated when an acoustic wave crosses the interface between the fluid and the thin porous disc, without interference of electrical fields that are present within seismic body waves. The predicted pressure wavefield agrees well with the measurements in terms of acoustic wave travel times, waveforms, and amplitudes. The electric wavefield predictions agree with the recordings in terms of travel times, waveforms, and spatial amplitude decay. A discrepancy in amplitude of the converted EM signal is observed. Theoretical amplitudes that are smaller than the measurements were also reported in previous literature. These results seem to validate seismoelectric theory.
\end{abstract}

Keywords Seismoelectric $\cdot$ Electrokinetic $\cdot$ Interface response $\cdot$ Acoustic $\cdot$ Electromagnetic

M. D. Schakel $(\varangle) \cdot$ D. M. J. Smeulders · E. C. Slob · H. K. J. Heller

Department of Geotechnology, Delft University of Technology,

PO Box 5048, 2600 GA, Delft, The Netherlands

e-mail: m.d.schakel@tudelft.nl

D. M. J. Smeulders

e-mail: d.m.j.smeulders@tudelft.nl

E. C. Slob

e-mail: e.c.slob@tudelft.nl

H. K. J. Heller

e-mail: h.k.j.heller@tudelft.nl

D. M. J. Smeulders

Department of Mechanical Engineering, Eindhoven University of Technology,

PO Box 513, 5600 MB, Eindhoven, The Netherlands

e-mail: d.m.j.smeulders@tue.nl 


\section{Introduction}

Grains of fluid-saturated porous media acquire bound surface charges that are balanced by a layer of mobile counter ions in the fluid. This ensemble of bound and mobile charge layers is referred to as the electric double layer and gives rise to coupled seismic and electromagnetic (EM) wave propagation. Two seismoelectric effects are distinguished in literature: (1) an electric field fixed to the seismic pulse (coseismic) travels with seismic wave velocity through a porous medium (e.g., Pride and Haartsen 1996) and (2) a seismic wave that traverses an interface with a contrast in mechanical and/or electrical properties produces independently traveling EM signals that propagate with the speed of a diffusive EM field (e.g., Pride and Haartsen 1996; Haartsen and Pride 1997). Following Haines and Pride (2006), we refer to the latter as the seismoelectric interface response.

The coseismic electric field and interface response effects are predicted by the full-waveform numerical model of Haartsen and Pride (1997). In their full-waveform simulations, Garambois and Dietrich (2002) find that the seismoelectric interface response is produced primarily within the first Fresnel zone of seismic to EM-wave conversion. Haines and Pride (2006) present a finite difference algorithm for seismoelectric wave propagation. These authors employ Pride's (1994) governing equations for coupled seismic and EM-wave propagation, which is also called electrokinetic theory. This theory combines poroelastic and EM-theory through the zeta-potential. This electric potential quantifies the amount of charge separation between the bound and mobile charge layers. The zeta-potential is comprised in Pride's frequency-dependent electrokinetic coupling coefficient, which is experimentally validated by Reppert et al. (2001) and Schoemaker (2011). The electrokinetic coupling coefficient couples electric and mechanical fields in extended Ohm's and Darcy's laws. These laws are called the transport equations. Alternative transport equations are proposed by Revil and Linde (2006) for microporous media. The coupling in Revil and Linde (2006) is based on the amount of excess electrical charge in the pore volume. Revil and Jardani (2010) extend the transport equations of Revil and Linde (2006) to porous media saturated with viscoelastic solvents. Revil and Jardani also present a finite element code with which they model coseismic and interface response fields due to boundaries between an oil-filled reservoir and an embedding medium. Jardani et al. (2010) use the relations of Revil and Linde (2006) in an inversion algorithm for poroelastic parameters and electrical conductivity, which uses seismic and seismoelectric data.

Coseismic and interface response fields are also measured in field and laboratory studies. Long and Rivers (1975) measure a change in voltage along the surface in a field study and attribute its cause to the Rayleigh and compressional waves. Thompson and Gist (1993) report on a field study in which conversion of seismic to EM energy at a depth of $300 \mathrm{~m}$ is observed. Butler et al. (1996) measure the seismoelectric conversion due to a shallow boundary, which is subsequently imaged. Dupuis et al. (2007) image the water table using seismoelectric interface responses. Zhu et al. (1999) measure the coseismic electrical field due to a Stoneley wave and interface responses in laboratory scale borehole models. Mikhailov et al. (2000) and Dupuis et al. (2009) measure these fields in field boreholes. Mikhailov et al. (2000) also propose a theoretical model to determine porosity and permeability from the Stoneley wave coseismic field. Zhu and Toksöz (2003) show that interface response fields in laboratory crosshole models are sensitive to fracture aperture and orientation. In a laboratory water column, filled with sediment in the lower part, Block and Harris (2006) measure the interface response field by electrodes in the fluid and the sediment as well as the coseismic fields within the sediments. Block and Harris match measured fast P-wave coseismic field amplitudes to plane-wave theoretical values as a function of Pride's dynamic conductivity. They find that 
additional (surface) conductivity is required to match the potentials for medium-grain sand sediment and that no additional conductivity is necessary for glass beads. In laboratory scale fluid-filled fractured boreholes, Zhu and Toksöz (2005) report on coseismic magnetic fields measured with a Hall-effect sensor and associate these fields with a Stoneley wave. Bordes et al. (2006) report on magnetic fields associated with shear waves, which are measured with induction magnetometers in a low magnetic noise environment in a fluid-filled sand column. Bordes et al. also assess the electrokinetic origin of the magnetic (and electric) effects, i.e., the signals are associated with the electric double layer that forms between the solid and the fluid electrolyte of a porous medium. Bordes et al. (2008) quantify the amplitude of the coseismic magnetic fields within the seismic shear waves.

Several researchers compare electrokinetic theory with measurements. Mikhailov et al. (1997) compare wavefield predictions of the seismoelectric interface response and refracted wavefield against field data. They find agreement in terms of arrival times and spatial dependency of seismoelectric amplitudes. Garambois and Dietrich (2001) perform field measurements of coseismic and interface response fields. Adopting plane-wave solutions of Pride's electrokinetic theory, they derive low-frequency transfer functions for coseismic fields and show that the transfer function theoretical predictions are consistent in waveform and amplitude with the field measurements. Haines et al. (2007) obtain a seismoelectric image from controlled field experiments and compare it with a synthetic (full-waveform) image. Both the coseismic and interface response fields are kinematically predicted by their model. Zhu et al. (2000) observe the coseismic and interface response fields in controlled laboratory experiments and find kinematic agreement with a full-waveform numerical model. They also confirm the electrokinetic nature of the signals. Schakel et al. (2011a) develop a full-waveform seismoelectric model based on the seismoelectric theory as derived by Pride (1994) and Pride and Haartsen (1996) and show that the model properly predicts laboratory measurements of coseismic and interface response fields in terms of travel time, waveform, polarity, amplitude, and spatial amplitude decay. In a similar way, Schakel et al. (2011b) compare full-waveform fluid/porous-medium interface response predictions as a function of salinity and distance to the interface. They find agreement in waveform and spatial amplitude pattern at all conductivities. They also find agreement in amplitudes at moderate salinity $\left(>\sim 10^{-3}\right.$ $\mathrm{M} \mathrm{NaCl})$, while the amplitudes are overpredicted at low salinity $\left(\sim 10^{-4} \mathrm{M} \mathrm{NaCl}\right)$.

It is often difficult to appropriately assess the full set of poroelastic, EM and electrokinetic parameters. In this article, we model the seismoelectric fluid/porous-medium interface response and compare the predictions with laboratory data. As in the works of Schakel et al. (2011a,b), our experimental setup allows to study the independently propagating EM signals that are generated when the acoustic wave crosses the interface between the fluid and the porous disc, without interference of the electrical fields within seismic body waves. We complement the studies of Schakel et al. $(2011 \mathrm{a}, \mathrm{b})$ by considering the EM signals that are generated when the porous sample is thin (with respect to the seismic wavelength). Porous media saturated with fluid electrolytes and wave propagation in the high-frequency regime is considered. We measured all parameters that are required for the seismoelectric numerical model (or they were known from literature). We start by introducing a model based on the Sommerfeld integral and the complete (frequency-dependent) seismoelectric theory of Pride. The model gives predictions of the fluid pressure and electric wavefields, which are subsequently compared with measurements of these fields. The modeled pressure wavefield agrees well with the measurements in terms of travel times, waveforms, and amplitudes. The modeled electric pulses agree with the recordings in terms of travel times, waveforms and spatial amplitude decay. However, the amplitudes are overestimated by theory. The latter observation is in agreement with previous findings. 


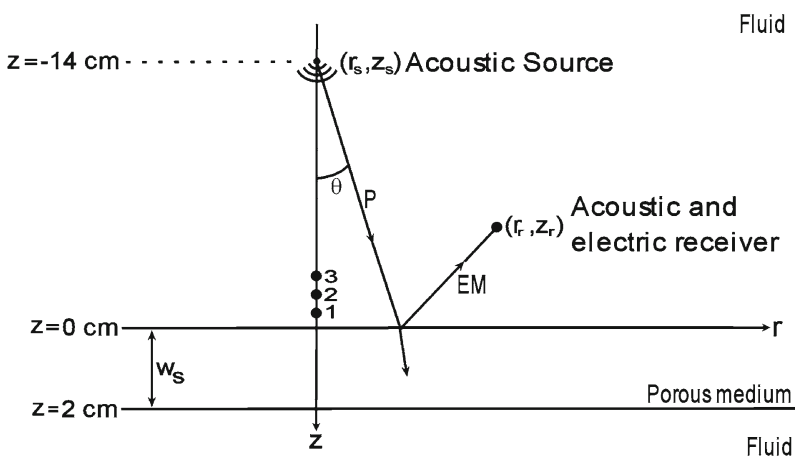

Fig. 1 Geometry of the seismoelectric model and experiments. Simultaneous measurements of pressure and electric potential are performed along the $z$-axis at $z=-1 \mathrm{~cm}$ (position 1 ), $z=-2 \mathrm{~cm}$ (position 2), and $z=-3 \mathrm{~cm}$ (position 3). A pressure $(\mathrm{P})$ wave is converted to an EM-wave at the interface. The total acoustic pressure and the total electric potential is given by integrals over complex-valued $\theta$ (Eqs. 7-8 and Eq. 9, respectively)

\section{Modeling of the Pressure and Electric Wavefields in the Fluid}

An isotropic, homogeneous porous medium is immersed in a compressible fluid such that horizontal interfaces are at $z=0$ and $z=2 \mathrm{~cm}$ (see Fig. 1). The acoustic source pressure is located in the region $z<0$ and modeled as a modified spherical wave source (e.g., Hall 1987):

$$
\hat{p}(\omega, R, \theta)=\frac{A(\omega) D(\theta)}{R} \exp [-i k R],
$$

where $\omega$ is the radial frequency, $R=\sqrt{\left(r-r_{\mathrm{S}}\right)^{2}+\left(z-z_{\mathrm{S}}\right)^{2}}$ is the distance to the source, $\theta$ is the angle of incidence (see Fig. 1), $A(\omega)$ is the amplitude spectrum, and $k$ is the fluid wavenumber. The directivity function $D(\theta)$, which characterizes the radiation pattern of the source, is given by:

$$
D(\theta)=\frac{J_{1}(k a \sin \theta)}{k a \sin \theta}
$$

Here, $J_{1}$ is the Bessel function of the first kind and first order and $a$ is the radius of the transducer. The modeled source pressure wavefield is expanded into conical waves by means of the Sommerfeld integral (see, for example, Brekhovskikh 1960; Aki and Richards 2002). The pressure wavefield that travels directly from the source to the receiver is then expressed as:

$$
\hat{P}_{\mathrm{d}}\left(\omega, r_{\mathrm{r}}, z_{\mathrm{r}}\right)=-i A(\omega) \int_{0}^{\infty} \frac{k_{\mathrm{r}}}{k_{\mathrm{z}}} D\left(k_{\mathrm{r}}\right) J_{0}\left(k_{\mathrm{r}} r_{\mathrm{r}}\right) \exp \left[-i k_{\mathrm{z}}\left|z_{\mathrm{r}}-z_{\mathrm{s}}\right|\right] \mathrm{d} k_{\mathrm{r}},
$$

where $k_{\mathrm{r}}=k \sin \theta$ and $k_{\mathrm{z}}=k \cos \theta$. The total pressure field in the fluid is composed of the direct and the reflected pressure wavefields. The latter consists of waves that are reflected from the interface at $z=0$ and so-called multiple waves $\left(r_{\mathrm{s}}=0 ; z_{\mathrm{s}}, z_{\mathrm{r}}<0\right)$ : 


$$
\hat{P}_{\mathrm{r}}\left(\omega, r_{\mathrm{r}}, z_{\mathrm{r}}\right)=-i A(\omega) \int_{0}^{\infty} \frac{k_{\mathrm{r}}}{k_{\mathrm{z}}} D\left(k_{\mathrm{r}}\right) J_{0}\left(k_{\mathrm{r}} r_{\mathrm{r}}\right) \exp \left[i k_{\mathrm{z}} z_{\mathrm{s}}\right]\left[R^{\mathrm{P}}\left(k_{\mathrm{r}}\right)+\mathrm{P}_{\mathrm{M}}\left(k_{\mathrm{r}}\right)\right] \exp \left[i k_{\mathrm{z}} z_{\mathrm{r}}\right] \mathrm{d} k_{\mathrm{r}}
$$

where $R^{\mathrm{P}}\left(k_{\mathrm{r}}\right)$ is the reflection coefficient. The reflected pressure associated with $N$ multiples is described by:

$$
P_{\mathrm{M}}\left(k_{\mathrm{r}}\right)=T^{\mathrm{Pf}}\left(k_{\mathrm{r}}\right) \sum_{j=1}^{N}\left(\exp \left[-2 i k_{\mathrm{z}}^{\mathrm{Pf}} w_{\mathrm{s}}\right]\right)^{j}\left(R^{\mathrm{Pf}}\left(k_{\mathrm{r}}\right)\right)^{2 j-1} T^{\mathrm{P}}\left(k_{\mathrm{r}}\right),
$$

where $T^{\mathrm{Pf}}\left(k_{\mathrm{r}}\right)$ denotes the transmission to a (fast) compressional (Pf-) wave into the porous medium due to the incident acoustic wave, $w_{\mathrm{S}}$ is the width of a porous sample and $k_{\mathrm{z}}^{\mathrm{Pf}}$ is the vertical component of the Pf-wavenumber. Internal reflection of a Pf-wave is described by $R^{\mathrm{Pf}}\left(k_{\mathrm{r}}\right)$ and $T^{\mathrm{P}}\left(k_{\mathrm{r}}\right)$ denotes the transmission of an acoustic wave into the fluid due to an incident Pf-wave from the porous medium. Equation 5 describes Pf-waves that bounce back and forth between the interfaces at $z=0$ and $z=2 \mathrm{~cm}$, after which they are transmitted as pressure waves into the fluid. The simplification in the above model is that generated Biot slow waves decay to zero in their way to the next interface. This is a reasonable assumption, because the slow wave skin depth is approximately $1.8 \mathrm{~cm}$ at $100 \mathrm{kHz}$. Our approach is supported by Pride and Garambois (2002) who showed that all poroelastic wavemodes are required in the boundary value problem solution in order to properly model seismoelectric interface response fields. Only a limited amount of Pf multiples are significant as they lose energy at each reflection or transmission. Expressing $k_{\mathrm{r}}$ and $k_{\mathrm{z}}$ in terms of $k$ and $\theta$, Eq. 3 is written as:

$$
\hat{P}_{\mathrm{d}}\left(\omega, r_{\mathrm{r}}, z_{\mathrm{r}}\right)=-i A(\omega) \int_{0}^{\pi / 2+i \infty} D(\theta) k \sin \theta J_{0}\left(k r_{\mathrm{r}} \sin \theta\right) \exp \left[-i k\left|z_{\mathrm{r}}-z_{\mathrm{s}}\right| \cos \theta\right] \mathrm{d} \theta .
$$

The path of integration is along straight lines from 0 to $\pi / 2$ and from $\pi / 2$ to $\pi / 2+i \infty$ in the complex $\theta$-plane. The second integral over complex $\theta$ is simplified further employing Eq. 2 and the substitution $\theta=\pi / 2+i \ln \left[\sqrt{\gamma^{2}+1}+\gamma\right]$ :

$$
\begin{aligned}
\hat{P}_{\mathrm{d}}\left(\omega, r_{\mathrm{r}}, z_{\mathrm{r}}\right)= & -\frac{i A(\omega)}{a} \int_{0}^{\pi / 2} J_{0}\left(k r_{\mathrm{r}} \sin \theta\right) J_{1}(k a \sin \theta) \exp \left[-i k\left|z_{\mathrm{r}}-z_{\mathrm{s}}\right| \cos \theta\right] \mathrm{d} \theta \\
& +\frac{A(\omega)}{a} \int_{0}^{\infty} J_{0}\left(k r_{\mathrm{r}} \sqrt{\gamma^{2}+1}\right) \frac{J_{1}\left(k a \sqrt{\gamma^{2}+1}\right)}{\sqrt{\gamma^{2}+1}} \exp \left[-k\left|z_{\mathrm{r}}-z_{\mathrm{s}}\right| \gamma\right] \mathrm{d} \gamma .
\end{aligned}
$$

The reflected pressure is written in a similar way as Eq. 7:

$$
\begin{aligned}
\hat{P}_{\mathrm{r}}= & -\frac{i A}{a} \int_{0}^{\pi / 2} J_{0} J_{1} \exp \left[i k z_{\mathrm{s}} \cos \theta\right]\left[R^{\mathrm{P}}+P_{\mathrm{M}}\right] \exp \left[i k z_{\mathrm{r}} \cos \theta\right] \mathrm{d} \theta \\
& +\frac{A}{a} \int_{0}^{\infty} J_{0} \frac{J_{1}}{\sqrt{\gamma^{2}+1}} \exp \left[k z_{\mathrm{s}} \gamma\right]\left[R^{\mathrm{P}}+P_{\mathrm{M}}\right] \exp \left[k z_{\mathrm{r}} \gamma\right] \mathrm{d} \gamma,
\end{aligned}
$$



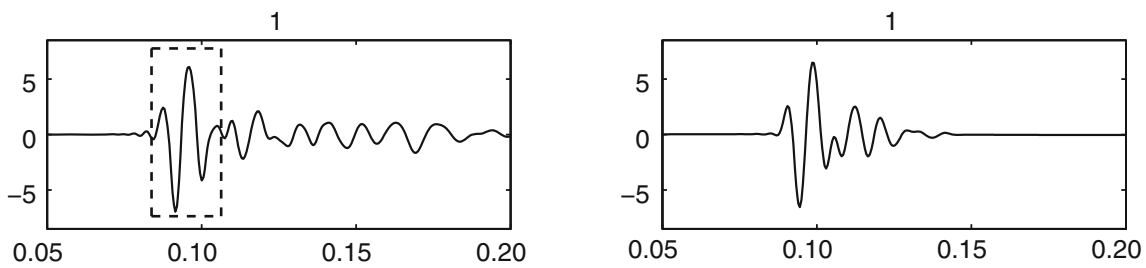

2

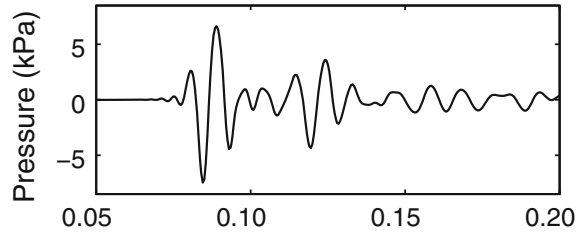

3

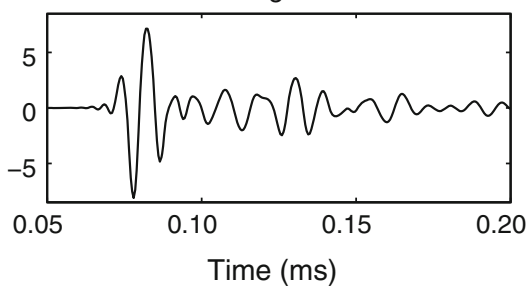

2

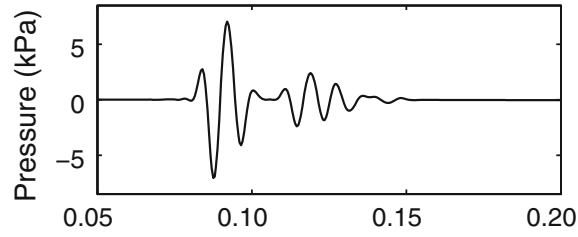

3

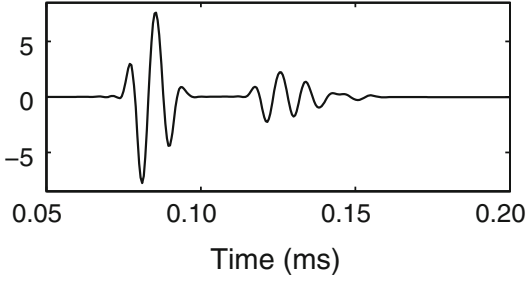

Fig. 2 Measured (left) and modeled (right) pressure within the fluid at positions 1-3 of Fig. 1. The dashed rectangle indicates the input pressure pulse employed in the model

where the dependencies are now implicitly assumed. We use a recursive adaptive Simpson quadrature algorithm implemented in Matlab to evaluate Eqs. 7-8 for each frequency. Timedomain expressions are obtained through an inverse fast Fourier transformation for positions $1-3$ in Fig. 1. The experimentally recorded incident pressure waveform at $(r, z)=(0,-1)$, indicated by the dashed rectangle in the upper left plot of Fig. 2, is used as input spectrum $A(\omega)$ and a $40-170 \mathrm{kHz}$ numerical band-pass filter is applied in the simulations. The input parameters of Table 1 are used and two multiples are taken into account ( $N=2$ in Eq. 5). Figure 2 (right) shows the resulting modeled pressure. The first pulses at positions 1-3 arrive at $87.6,80.8$, and $74.1 \mu \mathrm{s}$, respectively. These arrival times correspond to the travel times of the acoustic wave from the source to the receiver position. The second pulse arrives first at the receiver position closest to the fluid/porous-medium interface and its travel time increases for receivers further from the interface. This pulse is the reflected pressure that consists of interfering reflected and multiple waves. Clearly, most of the Pf-wave energy is lost after it has bounced back and forth two times.

The total electric wavefield in the fluid consists of the reflected electric potential only, because electromagnetism is uncoupled from mechanical fields in the fluid. Therefore, we can write for the electric potential wavefield in the fluid $\left(r_{\mathrm{s}}=0 ; z_{\mathrm{s}}, z_{\mathrm{r}}<0\right)$ :

$$
\hat{V}=-\frac{i A}{a} \int_{0}^{\pi / 2} J_{0} J_{1} \exp \left[i k z_{\mathrm{s}} \cos \theta\right] R^{\mathrm{E}} \exp \left[i k_{\mathrm{z}}^{\mathrm{E}} z_{\mathrm{r}}\right] \mathrm{d} \theta
$$


Table 1 Parameters of the fluid and the porous medium

\begin{tabular}{|c|c|c|c|c|}
\hline Bulk modulus skeleton grains ${ }^{\mathrm{a}}$ & 50 & & & $\times 10^{9} \mathrm{~Pa}$ \\
\hline Bulk modulus (pore) fluid ${ }^{b}$ & 2.2 & & & $\times 10^{9} \mathrm{~Pa}$ \\
\hline Pore fluid viscosity ${ }^{\mathrm{b}}$ & 1 & & & $\times 10^{-3} \mathrm{~Pa} \mathrm{~s}$ \\
\hline (Pore) fluid density ${ }^{\mathrm{b}}$ & 1.000 & & & $\times 10^{3} \mathrm{~kg} / \mathrm{m}^{3}$ \\
\hline Relative permittivity (pore) fluid ${ }^{b}$ & 80 & & & - \\
\hline Relative permittivity solid ${ }^{\mathrm{b}}$ & 4.0 & & & - \\
\hline Weighted pore volume-to-surface ratio ${ }^{c}$ & 1.2 & & & $\times 10^{-5} \mathrm{~m}$ \\
\hline Bulk modulus framework of grains ${ }^{\mathrm{d}}$ & 6.6 & & & $\times 10^{9} \mathrm{~Pa}$ \\
\hline Shear modulus framework of grains ${ }^{\mathrm{d}}$ & 5.5 & & & $\times 10^{9} \mathrm{~Pa}$ \\
\hline (Pore) fluid conductivity ${ }^{\mathrm{e}}$ & 1.32 & & & $\times 10^{-3} \mathrm{~S} / \mathrm{m}$ \\
\hline Zeta-potential $^{\mathrm{f}}$ & -5.17 & & & $\times 10^{-2} \mathrm{~V}$ \\
\hline Porosity & 0.345 & & & - \\
\hline Solid density & 2.212 & & & $\times 10^{3} \mathrm{~kg} / \mathrm{m}^{3}$ \\
\hline Permeability & 3.1 & & & $\times 10^{-12} \mathrm{~m}^{2}$ \\
\hline Tortuosity & 2.1 & & & - \\
\hline Sample width & 2 & & & $\times 10^{-2} \mathrm{~m}$ \\
\hline Pf-wave complex velocity & $(3.239,0.008)$ & $(3.242,0.006)$ & $(3.243,0.005)$ & $\times 10^{3} \mathrm{~m} / \mathrm{s}$ \\
\hline Ps-wave complex velocity & $(8.03,0.88)$ & $(8.26,0.62)$ & $(8.37,0.51)$ & $\times 10^{2} \mathrm{~m} / \mathrm{s}$ \\
\hline S-wave complex velocity & $(1820,0.015)$ & $(1.824,0.011)$ & $(1827,0.009)$ & $\times 10^{3} \mathrm{~m} / \mathrm{s}$ \\
\hline EM-wave complex velocity & $(2.592,2.106)$ & $(3.816,2.541)$ & $(4.708,2.606)$ & $\times 10^{7} \mathrm{~m} / \mathrm{s}$ \\
\hline
\end{tabular}

These parameters are literature values (see superscripts) or obtained from independent measurements on the porous sample of the experiments. When applicable, fluid parameters are the same as for the porous medium. The temperature is $293.15 \mathrm{~K}$ and the magnetic permeability is $4 \pi \times 10^{-7} \mathrm{H} / \mathrm{m}$. Corresponding real (first number) and imaginary (second number) parts of the poroelastic wave speeds for the fast (Pf) and slow (Ps) compressional waves, the shear (S-) wave and the EM-wave are given at $50 \mathrm{kHz}$ (first column), $100 \mathrm{kHz}$ (second column), and $150 \mathrm{kHz}$ (third column)

a Johnson and Plona (1982)

b Lide (2010)

c Pride (1994); Johnson et al. (1987, 1994); Jocker and Smeulders (2009)

d van der Lee (2003)

e Conductivity is due to $10^{-4} \mathrm{M} \mathrm{NaCl}$ electrolyte and measured in the fluid surrounding the porous medium

f Obtained from Zetasizer (Malvern Instruments Ltd., Zetasizer Nano ZS) measurement on crushed porous sample suspensions in $10^{-4} \mathrm{M} \mathrm{NaCl}$. See Schakel et al. (2011b)

$$
+\frac{A}{a} \int_{0}^{\infty} J_{0} \frac{J_{1}}{\sqrt{\gamma^{2}+1}} \exp \left[k z_{\mathrm{s}} \gamma\right] R^{\mathrm{E}} \exp \left[i k_{\mathrm{z}}^{\mathrm{E}} z_{\mathrm{r}}\right] \mathrm{d} \gamma,
$$

where $R^{\mathrm{E}}$ is the seismoelectric reflection coefficient that describes the conversion of an acoustic to an EM-wave (Schakel and Smeulders 2010), and where the vertical component of the fluid EM-wavenumber is denoted by $k_{\mathrm{z}}^{\mathrm{E}}=\omega \sqrt{1 / c_{\mathrm{E}}^{2}(\omega)-\sin ^{2} \theta / c_{\mathrm{P}}^{2}}$ with $\operatorname{Im}\left[k_{\mathrm{z}}^{\mathrm{E}}(\theta)<0\right]$ and with $c_{\mathrm{E}}(\omega)$ and $c_{\mathrm{P}}$ the fluid EM and pressure wave velocities, respectively. Note that Eq. 9 only takes electric potential converted from $z=0$ into account, i.e., no seismoelectric effects associated with multiples are considered. We expect that this assumption will not alter the order of magnitude of the signal, although Pride and Garambois (2005) compute that for very 

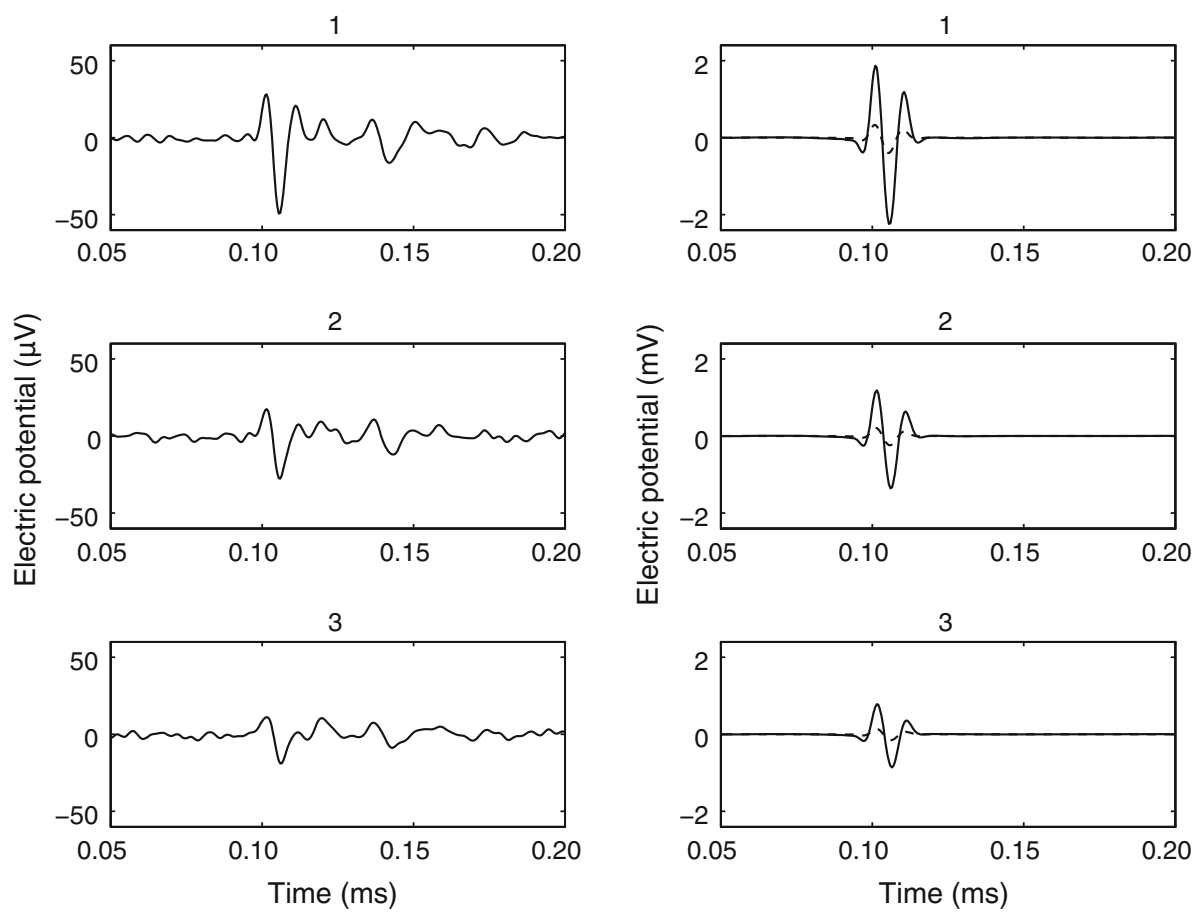

Fig. 3 Measured (left) and modeled (right) electric potential within the fluid at positions 1-3 of Fig. 1. The solid and dashed curves in the right panels correspond with the parameters of Table 1 and a modified version of this set (see text), respectively

thin (compared to the seismic wavelength) and low permeability layers, amplitudes increase by roughly a factor of 10 .

Equation 9 is evaluated in a similar way as Eqs. 7-8. The solid curves in Fig. 3 (right) show the resulting predicted electric potential waveforms. A pulse arrives at around $94 \mu \mathrm{s}$ for each position. This travel time corresponds with the acoustic travel time from the source to the fluid/porous-medium interface at $z=0$ (see Fig. 1). An EM signal is created when the acoustic wave impinges the porous-medium surface. The fluid EM-wave travels at large speed $\left(\sim 1.5 \times 10^{7} \mathrm{~m} / \mathrm{s}\right)$ so that the pulses arrive almost simultaneously at each position. Note that the pulse decays in amplitude as the distance from the interface increases (positions 1-3 in Fig. 1). The spatial dependence of the seismoelectric conversion at interfaces was documented extensively in literature (e.g., Thompson and Gist 1993; Butler et al. 1996; Haartsen and Pride 1997; Mikhailov et al. 1997; Garambois and Dietrich 2001, 2002; Haines and Pride 2006; Haines et al. 2007; Dupuis et al. 2007, 2009; Schakel et al. 2011b), although most of it considered spatial dependence parallel, rather than perpendicular, to the interface.

\section{Experiments}

\subsection{Seismoelectric Experimental Setup}

The experimental setup consists of a $58 \times 39 \times 28 \mathrm{~cm}$ water tank in which an acoustic wave source transducer (Panametrics V3638), a receiver hydrophone (Brüel \& Kjær 8103), an electrode (A-M systems Inc., $180 \mu \mathrm{m} \mathrm{Ag/AgCl)} \mathrm{and} \mathrm{a} \mathrm{porous} \mathrm{disc} \mathrm{(Technoglas} \mathrm{glass} \mathrm{filter} \mathrm{P3)}$ 


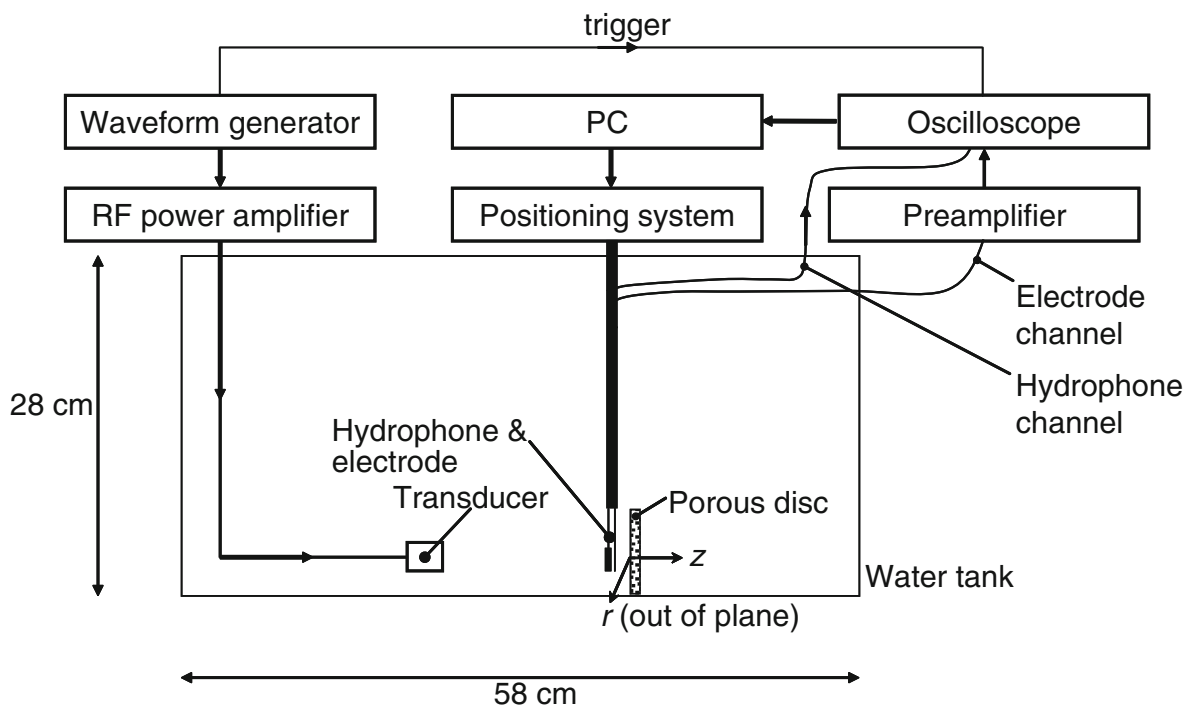

Fig. 4 Schematic of the experimental setup. Electrode potential recordings are with respect to ground level. The $r$ - and $z$-axes of Fig. 1 are also indicated

are installed (see Fig. 4). The sample is made of sintered glass (parameters listed in Table 1) and carefully saturated with and immersed in a $10^{-4} \mathrm{M} \mathrm{NaCl}$ electrolyte. A $100 \mathrm{kHz}$ single sine pulse (with a $1 \mathrm{~V}$ peak-to-peak amplitude) from a waveform generator (Agilent Technologies 33220A) is fed into an amplifier (ENI 2100L RF Power Amplifier) set at $50 \mathrm{~dB}$ gain. The output signal is coupled into the piezoelectric source transducer. The receiver hydrophone and electrode are connected to an oscilloscope (Yokogawa DL4200). A 40-170 kHz numerical band-pass filter is applied in all measurements. The geometry of the experiment is depicted in Fig. 1. At positions 1-3 along the $z$-axis, pressure and electric potential measurements were performed simultaneously. Electric potentials were amplified 40 times (Analogic D1000). No electric field is created but by conversion from the interfaces. Therefore, the experimental geometry allows to study the independently propagating EM signals that are generated when the acoustic waves cross the interfaces, without interference of body wave coseismic fields. The porous disc is thin with respect to the Pf-wave wavelength (see Table 1).

\subsection{Pressure and Electric Measurements}

The pressure on-axis measurements are averaged over 8192 source pulses and shown in Fig. 2 (left). After the direct pressure waves arrive, a complex pressure signal is received. The measured pressure of the direct and reflected wavefields agree with the modeled pressure in terms of amplitude, travel times and waveforms. However, not all pressure signals are predicted by the model.

The true amplitude electric potential on-axis measurements are averaged over 8192 source pulses and shown in Fig. 3 (left). An electric pulse arrives at around $94 \mu$ s for each position. Thereafter a complex electric signal is recorded. The modeled first pulses agree with the measurements in terms of arrival times, waveforms and the relative amplitude decay perpendicular to the interface at $z=0$ (positions 1-3 in Fig. 1). Note that the amplitudes differ. Schakel et al. (2011b) also found that theoretical interface response amplitudes were overpredicted by their model at a $10^{-4} \mathrm{M} \mathrm{NaCl}$ salinity. The measured complex pattern after the first arrival 
is not predicted by the model. It is expected that additional parts of the recorded potentials can be explained by incorporating seismoelectric multiple wave propagation effects. From the Pf-wave speed (see Table 1) and the thickness of the sample $(2 \mathrm{~cm})$, we conclude that a second interface response pulse generated at $z=2 \mathrm{~cm}$ will overlap in time with the first pulse that arrives at around $94 \mu \mathrm{s}$, i.e., it arrives approximately $6 \mu \mathrm{s}$ later, and therefore, will also generate electric signals up to $6 \mu$ s later. Schakel et al. (2011a) showed that by expanding Eq. 9 with appropriate seismoelectric and mechanical reflection and transmission coefficients and wave propagation terms, additional interface response fields that are generated by seismic waves that scatter within the sample can be described. However, as the Pf-wavelength $(\sim 3 \mathrm{~cm})$ exceeds the thickness of the sample $(\sim 2 \mathrm{~cm})$, we are dealing with a so-called thinlayer geometry, which makes the relation between the seismic pressure traces with interface response fields less straightforward. Explaining thin-layer seismoelectric responses by an appropriate seismoelectric model will be part of future research.

\subsection{Model Seismoelectric Amplitude Parameter Sensitivity}

The overpredicted seismoelectric model amplitudes can be attributed to uncertainties in the porous-medium parameters employed in the model. The fluid conductivity was measured in the fluid outside the pores of the porous sample. At low conductivity, this may result in an underestimated value of porous sample bulk conductivity (Block and Harris 2006), which results in modeled electric potentials that are larger than the measurements. Schakel and Smeulders (2010) show that the seismoelectric reflection coefficient $R^{\mathrm{E}}$ is highly sensitive to pore fluid conductivity. We investigate the model seismoelectric amplitude sensitivity to conductivity by incorporating an additional surface conductivity of $4.2 \times 10^{-8} \mathrm{~S}$, which is the value with which Block and Harris (2006) fitted bulk conductivity to pore fluid conductivity for medium-grain sand, into the model. We also arbitrarily change the pore fluid conductivity to $6.6 \times 10^{-3} \mathrm{~S} / \mathrm{m}$ (five times the initial value) and the zeta-potential to $-2 \times 10^{-2} \mathrm{~V}$ (see Schakel et al. (2011b) for a discussion on the zeta-potential measurement). The result is shown by the dashed curves in the right panels of Fig. 3. Indeed the simulated amplitudes are significantly diminished and fall within range of the measured amplitudes, although they are still overpredicted.

\section{Conclusions}

A seismoelectric model based on the Sommerfeld integral gave numerical predictions of fluid pressure and electric potential wavefields. The model considered a porous medium enclosed by fluid, in which an acoustic source was located. Simultaneous measurements of fluid pressure and electric potential were performed at different locations in a laboratory setup. The predicted and measured pressure wavefields agreed well. The seismoelectric interface response theoretical predictions and measurements agreed in terms of travel times, waveforms and spatial amplitude decay. A discrepancy in amplitude was observed, which was also found in previous reports. By changing key electrokinetic parameters, the simulated seismoelectric amplitudes were diminished and fell within range of the measured amplitudes. These results seem to validate seismoelectric theory. Although general agreement was found between the seismoelectric simulations and the measurements for the first arrival, complex patterns that were recorded afterward were not predicted by the model. In order to explain these patterns, the seismoelectric model must be expanded with multiple wave effects. However, the relation between the seismic pressure traces and interface response fields was not straightforward, 
because of the thin-layer geometry. Further research will be aimed at explaining thin-layer seismoelectric responses by an appropriate seismoelectric model.

Acknowledgments This research was funded as a Shell-FOM (Fundamental Research on Matter) project within the research program "The physics of fluids and sound propagation". MDS thanks Jolanda van Haagen for technical support and Zhenya Zhu, Gabriel Chao, Bobby Hak, Christiaan Schoemaker and Karel van Dalen for valuable discussions. We thank an anonymous reviewer for in-depth comments and André Revil for the discussion on electrokinetic theory.

Open Access This article is distributed under the terms of the Creative Commons Attribution Noncommercial License which permits any noncommercial use, distribution, and reproduction in any medium, provided the original author(s) and source are credited.

\section{References}

Aki, K., Richards, P.G.: Quantitative Seismology. University Science Books, Sausalito (2002)

Block, G.I., Harris, J.G.: Conductivity dependence of seismoelectric wave phenomena in fluid-saturated sediments. J. Geophys. Res. 111 (2006). doi:10.1029/2005JB003798

Bordes, C., Jouniaux, L., Dietrich, M., Pozzi, J.-P., Garambois, S.: First laboratory measurements of seismomagnetic conversions in fluid-filled Fontainebleau sand. Geophys. Res. Lett. 33 (2006). doi:10.1029/ 2005GL024582

Bordes, C., Jouniaux, L., Garambois, S., Dietrich, M., Pozzi, J.-P., Gaffet, S.: Evidence of the theoretically predicted seismo-magnetic conversion. Geophys. J. Int. 174, 489-504 (2008)

Brekhovskikh, L.M.: Waves in Layered Media. Academic press, Bristol (1960)

Butler, K.E., Russell, R.D., Kepic, A.W., Maxwell, M.: Measurement of the seismoelectric response from a shallow boundary. Geophysics 61, 1769-1778 (1996)

Dupuis, J.C., Butler, K.E., Kepic, A.W.: Seismoelectric imaging of the vadose zone of a sand aquifer. Geophysics 72, A81-A85 (2007)

Dupuis, J.C., Butler, K.E., Kepic, A.W., Harris, B.D.: Anatomy of a seismoelectric conversion: Measurements and conceptual modeling in boreholes penetrating a sandy acquifer. J. Geophys. Res. 114 (2009). doi:10. 1029/2008JB005939

Garambois, S., Dietrich, M.: Seismoelectric wave conversions in porous media: field measurements and transfer function analysis. Geophysics 66, 1417-1430 (2001)

Garambois, S., Dietrich, M.: Full waveform numerical simulations of seismoelectromagnetic wave conversions in fluid-saturated stratified porous media. J. Geophys. Res. 107 (2002). doi:10.1029/2001JB000316

Haartsen, M.W., Pride, S.R.: Electroseismic waves from point sources in layered media. J. Geophys. Res. 102, 24745-24769 (1997)

Haines, S.S., Pride, S.R.: Seismoelectric numerical modeling on a grid. Geophysics 71, N57-N65 (2006)

Haines, S.S., Pride, S.R., Klemperer, S.L., Biondi, B.: Seismoelectric imaging of shallow targets. Geophysics 72, G9-G20 (2007)

Hall, D.E.: Basic Acoustics. Wiley, Singapore (1987)

Jardani, A., Revil, A., Slob, E., Söllner, W.: Stochastic joint inversion of 2D seismic and seismoelectric signals in linear poroelastic materials: a numerical investigation. Geophysics 75, N19-N31 (2010)

Jocker, J., Smeulders, D.: Ultrasonic measurements on poroelastic slabs: determination of reflection and transmission coefficients and processing for Biot input parameters. Ultrasonics 49, 319-330 (2009)

Johnson, D.L., Plona, T.J.: Acoustic slow waves and the consolidation transition. J. Acoust. Soc. Am. 72, 556$565(1982)$

Johnson, D.L., Koplik, J., Dashen, R.: Theory of dynamic permeability and tortuosity in fluid-saturated porous media. J. Fluid Mech. 176, 379-402 (1987)

Johnson, D.L., Hemmick, D.L., Kojima, H.: Probing porous media with first and second sound. I. Dynamic permeability. J. Appl. Phys. 76, 104-114 (1994)

Lide, D.R. (ed.): CRC Handbook of Chemistry and Physics, 90th Edn. (Internet Version 2010). CRC Press/Taylor and Francis. http://www.hbcpnetbase.com/ (2010). Accessed 30 Aug 2010

Long, L.T., Rivers, W.K.: Field measurement of the electroseismic response. Geophysics 40, 233-245 (1975)

Mikhailov, O.V., Haartsen, M.W., Toksöz, M.N.: Electroseismic investigation of the shallow subsurface: field measurements and numerical modeling. Geophysics 62, 97-105 (1997)

Mikhailov, O.V., Queen, J., Toksöz, M.N.: Using borehole electroseismic measurements to detect and characterize fractured (permeable) zones. Geophysics 65, 1098-1112 (2000) 
Pride, S.: Governing equations for the coupled electromagnetics and acoustics of porous media. Phys. Rev. B 50, 15678-15696 (1994)

Pride, S.R., Garambois, S.: Electroseismic wave theory of Frenkel and more recent developments. J. Eng. Mech. 131, 898-907 (2005)

Pride, S.R., Haartsen, M.W.: Electroseismic wave properties. J. Acoust. Soc. Am. 100, 1301-1315 (1996)

Pride, S.R., Garambois, S.: The role of Biot slow waves in electroseismic wave phenomena. J. Acoust. Soc. Am. 111, 697-706 (2002)

Reppert, P.M., Morgan, F.D., Lesmes, D.P., Jouniaux, L.: Frequency-dependent streaming potentials. J. Colloid Interface Sci. 234, 194-203 (2001)

Revil, A., Jardani, A.: Seismoelectric response of heavy oil reservoirs: theory and numerical modelling. Geophys. J. Int. 180, 781-797 (2010)

Revil, A., Linde, N.: Chemico-electromechanical coupling in microporous media. J. Colloid Interface Sci. 302, 682-694 (2006)

Schakel, M., Smeulders, D.: Seismoelectric reflection and transmission at a fluid/porous-medium interface. J. Acoust. Soc. Am. 127, 13-21 (2010)

Schakel, M.D., Smeulders, D.M.J., Slob, E.C., Heller, H.K.J.: Laboratory measurements and theoretical modeling of seismoelectric interface response and coseismic wave fields. J. Appl. Phys. 109, 074903 (2011a). doi:10.1063/1.3567945

Schakel, M.D., Smeulders, D.M.J., Slob, E.C., Heller, H.K.J.: Seismoelectric interface response: experimental results and forward model. Geophysics 76, N29-N36 (2011b)

Schoemaker, F.C.: Electrokinetic conversion. PhD thesis, Delft University of Technology (2011)

Thompson, A.H., Gist, G.A.: Geophysical applications of electrokinetic conversion. Lead. Edge 12, 11691173 (1993)

van der Lee, C.J.: A comprehensive study of transmission and reflection phenomena in water-saturated porous slabs. M.Sc. thesis, Delft University of Technology (2003)

Zhu, Z., Haartsen, M.W., Toksöz, M.N.: Experimental studies of electrokinetic conversions in fluid-saturated borehole models. Geophysics 64, 1349-1356 (1999)

Zhu, Z., Haartsen, M.W., Toksöz, M.N.: Experimental studies of seismoelectric conversions in fluid-saturated porous media. J. Geophys. Res. 105, 28055-28064 (2000)

Zhu, Z., Toksöz, M.N.: Crosshole seismoelectric measurements in borehole models with fractures. Geophysics 68, 1519-1524 (2003)

Zhu, Z., Toksöz, M.N.: Seismoelectric and seismomagnetic measurements in fractured borehole models. Geophysics 70, F45-F51 (2005) 